\title{
Effect of Ashwagandharishta on the lipid profile of male and female rats
}

\author{
Tasmina Rahman ", Mohammad Salahuddin Bhuiya, Rakib Hasan and \\ M. S. K. Choudhuri \\ Department of Pharmacy, Jahangirnagar University, Savar, Dhaka- 1342, Bangladesh.
}

\begin{abstract}
The effect of Ashwagandharishta on the lipid profile and chronic toxicity of both male and female Albino rats were observed for 51 days. Our results demonstrate that the treatment of the plant extract failed to exhibit any statistically significant change (increase/ decrease) in the serum cholesterol (Total), high density lipoprotein (HDL), low density lipoprotein (LDL) and triglyceride of the male rats. But the female rats showed highly $(\mathrm{p}<0.01)$ and very highly $(\mathrm{p}<0.001)$ significant decrease in the serum cholesterol (Total) levels of the medium and high dose treated groups respectively. Regarding HDL, ASG showed statistically significant decrease at low $(p<0.05)$, medium $(\mathrm{p}<0.01)$ and high $(\mathrm{p}<0.001)$ dose treated female rats. The LDL level of the medium dose treated female rats were statistically significant $(p<0.05)$. The serum Triglyceride decreased significantly at low $(\mathrm{p}<0.01)$ and high $(p<0.05)$ dose treated female rats.
\end{abstract}

Keywords: Ashwagandharishta, Serum Cholesterol, HDL, LDL and Triglyceride.

\section{INTRODUTION}

Cholesterol is a fat (also called a lipid) that is essential for life. It forms the membranes for cells in all organs and tissues in body. Cholesterol is also used for the production of steroid hormones, bile acids, and Vitamin D. A small amount of body's cholesterol circulates in the blood in complex particles called lipoproteins. These lipoproteins include some particles that carry excess cholesterol away for disposal (HDL-C, good cholesterol) and some particles that deposit cholesterol in tissues and organs (LDL-C, bad cholesterol). The cholesterol test is used to measure total cholesterol (good and bad) that is carried in the blood by lipoproteins. Cholesterol test is used to diagnose or monitor a disease but is used to estimate risk of developing a disease - specifically heart disease (Semenkovich, 2011; Richmond, 1992).

World Health Organization (WHO) reported that a substantial number of world population use herbal medicines for some aspect of primary health care. Because of the perception of low side effect profiles, traditional medicines now a days is of special research interest. In this study, Ashwagandharishta was used which is a classical ayurvedic formulation used in the treatment of Murchha (syncope), Mandagni (poor digestive power), etc. Ashwagandha (Withania somnifera D.), the key component of Ashwagandharishta (Table 1), (Anonymous, 1992), has anti-stress and anxiolytic activities. Further, it also affects brain-derived neurotrophic factor (BDNF) that boosts synaptic plasticity, delivers neuro-protection, augments neurotransmission, and has antidepressant effects (Tanna et al., 2012). It also has many actions on body like anti-

\footnotetext{
* Corresponding author. Email: tamirahman@yahoo.com
} 
ageing, adaptogenic, immune-modulatory, cardiovascular protection, hypothyroidism etc. (Kushwaha \& Karanjekar, 2011). Withania somnifera, also recognized as Indian ginseng, or winter cherry, has been an important herb in the Ayurvedic and indigenous medical systems. The roots of the plant are categorised as Rasayanas which promote health and longevity by enhancing defenses against disease, arresting the ageing process, revitalizing the body in debilitated conditions. It contains alkaloids (withanine, withasomnine), steroidal lactones and glycosides (withanolides \& sitoindosides). It is also used as a general tonic, to increase energy and improve health and longevity. Some studies suggest that, it may promote growth in children and improve hemoglobin level, red blood cell count, and physical performance in adults (Sandhu et al., 2010). It also increases heart weight and glycogen in myocardium and liver representing intensification of the anabolic process and enhances the duration of contractility as well as coagulation time (Kushwaha et al., 2012).

Table 1. Botanical name of ingredients along with the amount used to prepare Ashwagandharishta (ASG)

\begin{tabular}{c|c|c|c}
\hline No & Sanskrit Name & Botanical Name & Amount of ingredient \\
\hline 1 & Ashwagandha & Withania somnifera & $2.400 \mathrm{~kg}$ \\
2 & Sweta Musli & Asparagus adscendens & $960 \mathrm{~g}$ \\
3 & Manjishtha & Rubia cordifolia & $480 \mathrm{~g}$ \\
4 & Hareetaki & Terminalia chebula & $480 \mathrm{~g}$ \\
5 & Haridra & Curcuma longa & $480 \mathrm{~g}$ \\
6 & Daruharidra & Berberis aristata & $480 \mathrm{~g}$ \\
7 & Yashtimadhu & Glycyrrhiza glabra & $480 \mathrm{~g}$ \\
8 & Rasna & Pluchea lanceolata & $480 \mathrm{~g}$ \\
9 & Vidarikanda & Pueraria tuberose & $480 \mathrm{~g}$ \\
10 & Arjun Tvak & Terminalia arjuna & $480 \mathrm{~g}$ \\
11 & Mustaka & Cyperus rotundus & $480 \mathrm{~g}$ \\
12 & Trivrit & Ipomoea turpethum & $480 \mathrm{~g}$ \\
13 & Anantamool & Hemidesmus indicus & $384 \mathrm{~g}$ \\
14 & Krishna Sariva & Cryptolepis buchanan & $384 \mathrm{~g}$ \\
15 & Rakta Chandan & Pterocarpus santalinus & $384 \mathrm{~g}$ \\
16 & Chandan & Santalum album & $384 \mathrm{~g}$ \\
17 & Vacha & Acorus calamu & $384 \mathrm{~g}$ \\
18 & Chitrak Mool & Plumbago zeylanica & $384 \mathrm{~g}$ \\
19 & Water for decoction & 98.304 L reduced to & $12.288 \mathrm{~L}$ \\
20 & Dhataki puspa & Woodfordia fruticosa & $768 \mathrm{~g}$ \\
21 & Madhu & Honey & $14.400 \mathrm{~kg}$ \\
22 & Shunthi & Zingiber officinale & $96 \mathrm{~g}$ \\
23 & Maricha & Piper nigrum & $96 \mathrm{~g}$ \\
24 & Pippali & Piper longum & $96 \mathrm{~g}$ \\
25 & Tvak & Cinnamomum zeylanicum & $192 \mathrm{~g}$. \\
26 & Tejpata & Cinnamomum tamala & $192 \mathrm{~g}$. \\
27 & Elach & Elettaria cardamomum & $192 \mathrm{~g}$. \\
28 & Priyangu & Callicarpa macrophylla & $192 \mathrm{~g}$. \\
29 & Nagakeshar & Mesua ferrae & $96 \mathrm{~g}$. \\
\hline
\end{tabular}


To execute further clinical studies with this liquid preparation, this study was performed to evaluate the effects of ASG on the kidney function tests utilizing laboratory animals of both sexes.

\section{MATERIALS AND METHODS}

Drugs, chemicals and reagents: For the toxicological study, Ashwagandharishta was collected from Sri Kundeswari Aushadhalaya Limited, Chittagong. Ketamine injection was obtained from ACI Pharmaceuticals Limited, Bangladesh. All other reagents, assay kits and chemicals used in this research work were obtained from Human $\mathrm{GmbH}$, Wiesbaden, Germany.

Experimental Animals: Albino rats (Rattus novergicus : Sprague-Dawley strain, 48 weeks old, 70-80 g) of both sexes bred and maintained at the animal house of the Department of Pharmacy, Jahangirnagar University, were used in this toxicological experiment. They were kept in a well-ventilated hygienic experimental animal house under constant environmental and adequate nutritional conditions throughout the period of the experiment. They were fed with rat chow which was prepared according to the formula developed by Bangladesh Council of Scientific and Industrial Research (BCSIR). Water was given ad libitum and the animals maintained at 12 hours day and 12 hours night cycle. All experiments on rats were carried out in absolute compliance with the ethical guide for care and use of laboratory animals approved by Biosafety, Biosecurity and Ethical Committee of Faculty of Biological Sciences, Jahangirnagar University.

\section{Experimental design}

Acute toxicity study: The acute oral toxicity test was performed for Ashwagandharishta (OECD Guideline, 2000). Sixteen male \& female rats (70-80 g body weight) were divided into four groups of four animals each. Different doses $(1000 \mathrm{mg} / \mathrm{Kg}, 2000 \mathrm{mg} / \mathrm{Kg}, 3000$ $\mathrm{mg} / \mathrm{Kg}$ and $4000 \mathrm{mg} / \mathrm{Kg}$ ) of experimental drug (ASG) were administered to them. The dose was divided into two fractions and given within 12 hours. Then all the experimental animals were observed for mortality and clinical signs of toxicity at 1, 2, 3 and 4 hours and thereafter once a day for the next three days following ASG administration.

Chronic toxicity studies: A total of 40 males and 40 female rats were randomly assigned to the four groups, namely group I (Control: water), group II-Low Dose $(0.625 \mathrm{ml} / \mathrm{kg} \mathrm{BW}$ of ASG), group III-Medium Dose (5.0 ml/kg BW of ASG), and group IV-High Dose (40.0 ml/kg BW of ASG) consisting of 10 males and 10 females in each group. The animals of control group were administered with distilled water only as per the same volume as the drug treated group for 51 days. Ayurvedic medicinal preparation was administered to the rats by intra-gastric syringe at fixed time daily soon after acclimatization. All the experiments were carried out in absolute compliance with the ethical guideline for care and use of laboratory animals.

Blood samples collection and preparation of serum: At the end of treatment period (51 days), after 18 hours fasting, blood samples were collected from post vena cava of the rats anaesthetizing with Ketamine ( $500 \mathrm{mg} / \mathrm{Kg}$ body, intra peritoneal). Blood samples were transferred into plain sample tubes immediately for serum generation. It was then 
centrifuged at 4,000 g for 10 minutes using Bench top centrifuge (MSE Minor, England). The supernatant plasma samples were collected using dry Pasteur pipette and stored in the refrigerator for further analyses. All analyses were completed within 12 hours of sample collection.

Biochemical studies: Serum samples were analyzed for Serum Cholesterol, HDL, LDL and Triglyceride using spectrophotometer (Vitros-250, Johnson \& Johnson) Random Access Multibatch Chemistry Analyzer (USA).

Statistical analysis: Data were expressed as Mean \pm SEM (Standard Error of the Mean). One-way ANOVA followed by Dunnett's multiple comparison was performed to analyze this data set. ${ }^{*} p<0.05,{ }^{*} \mathrm{p}<0.01$ and $* * * \mathrm{p}<0.001$ was considered statistically significant, highly significant and very highly significant respectively. Statistical programs used were SPSS (version 16, IBM software Inc, USA).

\section{RESULTS AND DISCUSSION}

Acute toxicity study: When the rats were treated with a high dose of Ashwagandharishta (4000 mg/kg body weight), the mortality rate was found to be zero. This suggests that LD50 value of ASG is greater than $4000 \mathrm{mg} / \mathrm{kg}$ body weight. Thus ASG may be considered safe at this dose when given as a oral dose.

\section{Chronic toxicity study}

Effect of ASG on Serum Cholesterol (Total) level of male $\&$ female rat: All the doses of ASG showed no statistically significant increase or decrease in serum Cholesterol (Total) level of the male rats. On the other hand, in case of female rat, the medium and high dose results of ASG showed highly and very highly statistically significant decrease respectively in serum Cholesterol (Total) level at medium dose (18.107\%**) and high dose $(24.306 \% * * *)$. The low dose level was decreased also (12.071\%) but the decrease was not statistically significant (Table 2 \& Fig. 1).

Table 2. The effect of ASG on the Serum Cholesterol (Total) level of laboratory animals (Male \& Female Rat) compared to their corresponding Control group after 51 days

\begin{tabular}{l|cc|c|c}
\hline \multirow{2}{*}{ Group } & \multicolumn{4}{|c}{ Serum Cholesterol (Total) mg/dL } \\
\cline { 2 - 5 } & \multicolumn{2}{|c}{ Male Rat } & \multicolumn{2}{c}{ Female Rat } \\
\cline { 2 - 5 } & Mean \pm SEM & \% of Change & Mean \pm SEM & \% of Change \\
\hline Control & $47.100 \pm 0.959$ & - & $61.300 \pm 2.507$ & \\
ASG $(0.625 \mathrm{ml} / \mathrm{kg})$ & $45.800 \pm 0.592$ & $\downarrow 2.760 \%$ & $53.900 \pm 3.778$ & $\downarrow 12.071 \%$ \\
ASG $(5.0 \mathrm{ml} / \mathrm{kg})$ & $56.333 \pm 5.338$ & $\uparrow 19.603 \%$ & $50.200 \pm 1.093 * *$ & $\downarrow 18.107 \%$ \\
ASG $(40.0 \mathrm{ml} / \mathrm{kg})$ & $48.200 \pm 1.547$ & $\uparrow 2.335 \%$ & $46.400 \pm 1.194 * * *$ & $\downarrow 24.306 \%$ \\
\hline
\end{tabular}

Values are presented as mean \pm SEM $(n=10)$. One-way ANOVA followed by Dunnett's multiple comparison was performed to analyze this data set. ${ }^{*} p<0.05$, $* * \mathrm{p}<0.01$ and $* * * \mathrm{p}<0.001$ was considered statistically significant, highly significant and very highly significant respectively. Values are self explanatory when compared against control. $\uparrow$ : increase, $\downarrow$ : decrease. 


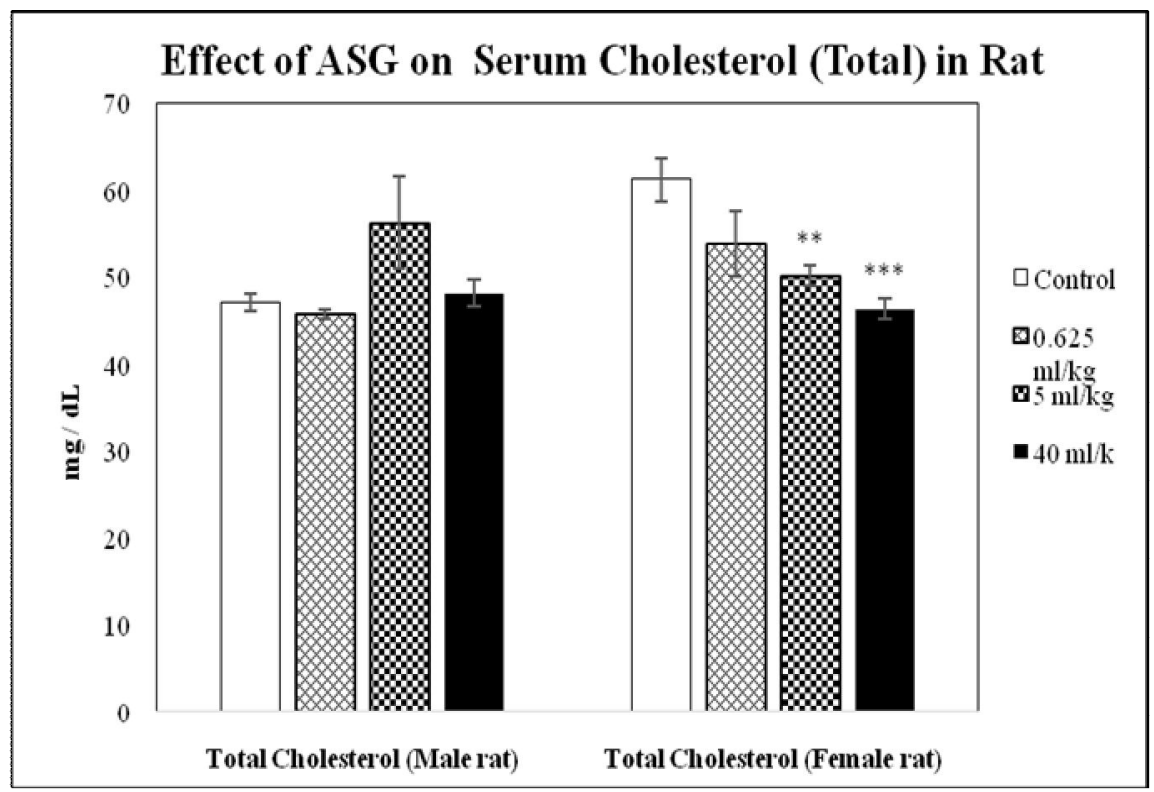

Fig. 1. The effect of ASG on the Serum Cholesterol (Total) level of laboratory animals (Male \& Female Rat) compared to their corresponding Control group after 51 days

Effect of ASG on Serum Cholesterol (HDL) level of male \& female rat: All the doses of ASG showed no statistically significant increase or decrease in serum Cholesterol (HDL) level of the male rats. On the other hand, in case of female rat, all the three dose results of ASG showed statistically significant decrease at low (12.000\%*), medium $(15.500 \% * *)$ and high dose $(20.500 \% * * *)$ (Table $3 \&$ Figure 2).

Table 3. The effect of ASG on the Serum Cholesterol (HDL) level of laboratory animals (Male \& Female Rat) compared to their corresponding Control group after 51 days

\begin{tabular}{|c|c|c|c|c|}
\hline \multirow[t]{3}{*}{ Group } & \multicolumn{4}{|c|}{ Serum Cholesterol (HDL) mg/dL } \\
\hline & \multicolumn{2}{|c|}{ Male Rat } & \multicolumn{2}{|c|}{ Female Rat } \\
\hline & Mean \pm SEM & $\%$ of Change & Mean \pm SEM & $\begin{array}{c}\% \text { of } \\
\text { Change }\end{array}$ \\
\hline Control & $36.000 \pm 0.977$ & - & $40.000 \pm 1.807$ & \\
\hline ASG $(0.625 \mathrm{ml} / \mathrm{kg})$ & $33.600 \pm 1.212$ & $\downarrow 6.666 \%$ & $35.200 \pm 0.928 *$ & $\downarrow 12.000 \%$ \\
\hline ASG $(5.0 \mathrm{ml} / \mathrm{kg})$ & $37.888 \pm 2.245$ & $\uparrow 5.246 \%$ & $33.800 \pm 0.813^{* *}$ & $\downarrow 15.500 \%$ \\
\hline ASG (40.0 ml/kg ) & $38.800 \pm 1.671$ & $\uparrow 7.777 \%$ & $31.800 \pm 1.263^{* * * *}$ & $20.500 \%$ \\
\hline
\end{tabular}

Values are presented as mean \pm SEM $(n=10)$. One-way ANOVA followed by Dunnett's multiple comparison was performed to analyze this data set. $* p<0.05$, $* * \mathrm{p}<0.01$ and $* * * \mathrm{p}<0.001$ was considered statistically significant, highly significant and very highly significant respectively. Values are self explanatory when compared against control. $\uparrow:$ increase, $\downarrow$ : decrease. 


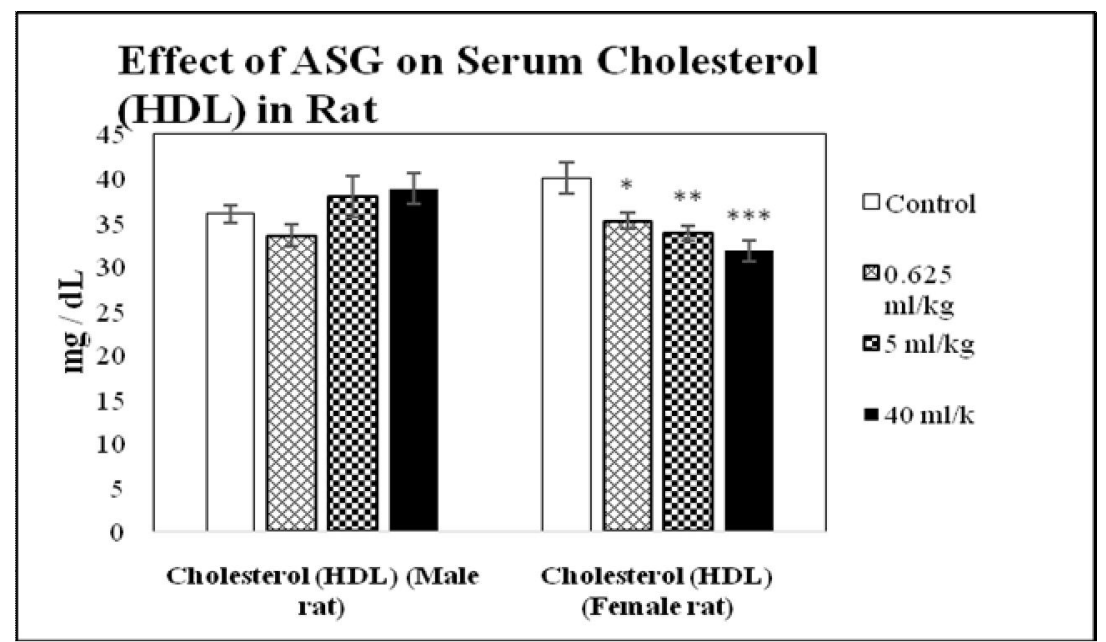

Fig. 2. The effect of ASG on the Serum Cholesterol (HDL) level of laboratory animals (Male \& Female Rat) compared to their corresponding Control group after 51 days

Effect of ASG on Serum Cholesterol (LDL) level of male \& female rat: LDL is a type of lipoprotein that carries cholesterol in the blood. LDL is considered to be undesirable because it deposits excess cholesterol in walls of blood vessel and contributes to hardening of the arteries (atherosclerosis) and heart disease. Hence LDL cholesterol is often termed "bad" cholesterol. The test for LDL cholesterol is used to predict risk of developing heart disease. Of all the forms of cholesterol in the blood, the LDL cholesterol is considered the most important form in determining risk of heart disease. LDL cholesterol is also used to monitor patients with prior Coronary heart disease, other atherosclerotic disease, or diabetes mellitus. Elevated levels of LDL cholesterol can indicate risk for heart disease (Rifai et al., 1992).

In this study, all the doses of both the male and female rats, ASG showed no statistically significant decrease in serum Cholesterol (LDL) level except the medium dose female rat result $(\downarrow 53.488 \%$ ), (Table $4 \&$ Figure 3 ).

Table 4. The effect of ASG on the Serum Cholesterol (LDL) level of laboratory animals (Male \& Female Rat) compared to their corresponding Control group after 51 days

\begin{tabular}{l|cc|c|c}
\hline \multirow{2}{*}{ Group } & \multicolumn{3}{|c}{ Serum Cholesterol (LDL) mg/dL } \\
\cline { 2 - 5 } & \multicolumn{2}{|c}{ Male Rat } & \multicolumn{2}{c}{ Female Rat } \\
\cline { 2 - 5 } & Mean \pm SEM & \% of Change & Mean \pm SEM & \% of Change \\
\hline Control & $1.620 \pm 0.722$ & - & $8.600 \pm 1.634$ & \\
ASG $(0.625 \mathrm{ml} / \mathrm{kg})$ & $0.200 \pm 1.592$ & $\downarrow 87.654 \%$ & $6.400 \pm 0.748$ & $\downarrow 25.581 \%$ \\
ASG $(5.0 \mathrm{ml} / \mathrm{kg})$ & $1.452 \pm 1.708$ & $\downarrow 10.339 \%$ & $4.000 \pm 0.977 *$ & $\downarrow 53.488 \%$ \\
ASG $(40.0 \mathrm{ml} / \mathrm{kg})$ & $1.488 \pm 1.100$ & $\downarrow 8.092 \%$ & $4.800 \pm 0.853$ & $\downarrow 44.186 \%$ \\
\hline
\end{tabular}

Values are presented as mean \pm SEM $(n=10)$. One-way ANOVA followed by Dunnett's multiple comparison was performed to analyze this data set. $* p<0.05$, **p $<0.01$ and $* * * \mathrm{p}<0.001$ was considered statistically significant, highly significant and very highly significant respectively. Values are self explanatory when compared against control. $\uparrow$ : increase, $\downarrow$ : decrease. 


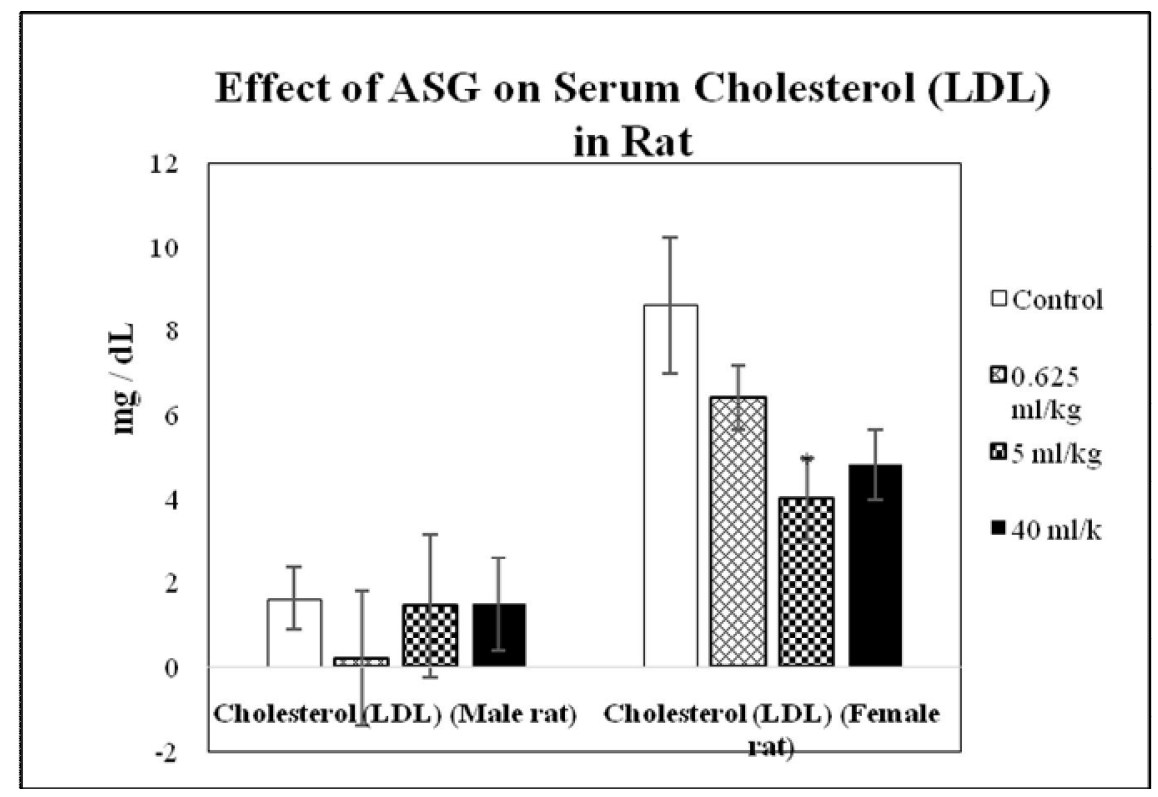

Fig. 3. The effect of ASG on the Serum Cholesterol (LDL) level utilizing of laboratory animals (Male \& Female Rat) compared to their corresponding Control group after 51 days

Effect of ASG on Serum Triglyceride (TG) level of male \& female rat: All the three different doses of ASG showed no statistically significant change in serum Triglyceride (TG) level for the male rat group. But a statistically significant decrease was observed for the low and high dose level in case of the female rat group (Table 5 \& Figure 4).

Table 5. The effect of ASG on the Serum Triglyceride (TG) level of laboratory animals (Male \& Female Rat) compared to their corresponding Control group after 51 days

\begin{tabular}{l|c|c|c|c}
\hline \multirow{2}{*}{ Group } & \multicolumn{3}{|c}{ Serum Triglyceride (TG) mg/dL } \\
\cline { 2 - 5 } & \multicolumn{2}{|c}{ Male Rat } & \multicolumn{2}{c}{ Female Rat } \\
\cline { 2 - 5 } & Mean \pm SEM & \% of Change & Mean \pm SEM & \% of Change \\
\hline Control & $61.988 \pm 3.477$ & - & $70.300 \pm 5.461$ \\
ASG $(0.625 \mathrm{ml} / \mathrm{kg})$ & $61.100 \pm 6.505$ & $\downarrow 1.433 \%$ & $48.000 \pm 4.550 * *$ & $\downarrow 31.721 \%$ \\
ASG $(5.0 \mathrm{ml} / \mathrm{kg})$ & $62.433 \pm 3.141$ & $\uparrow 0.716 \%$ & $62.400 \pm 5.493$ & $\downarrow 11.237 \%$ \\
ASG $(40.0 \mathrm{ml} / \mathrm{kg})$ & $61.120 \pm 4.937$ & $\downarrow 1.401 \%$ & $49.500 \pm 4.447 *$ & $\downarrow 29.587 \%$ \\
\hline
\end{tabular}

Values are presented as mean \pm SEM $(n=10)$. One-way ANOVA followed by Dunnett's multiple comparison was performed to analyze this data set. ${ }^{*} p<0.05$, **p $<0.01$ and $* * * \mathrm{p}<0.001$ was considered statistically significant, highly significant and very highly significant respectively. Values are self explanatory when compared against control. $\uparrow$ : increase, $\downarrow$ : decrease. 


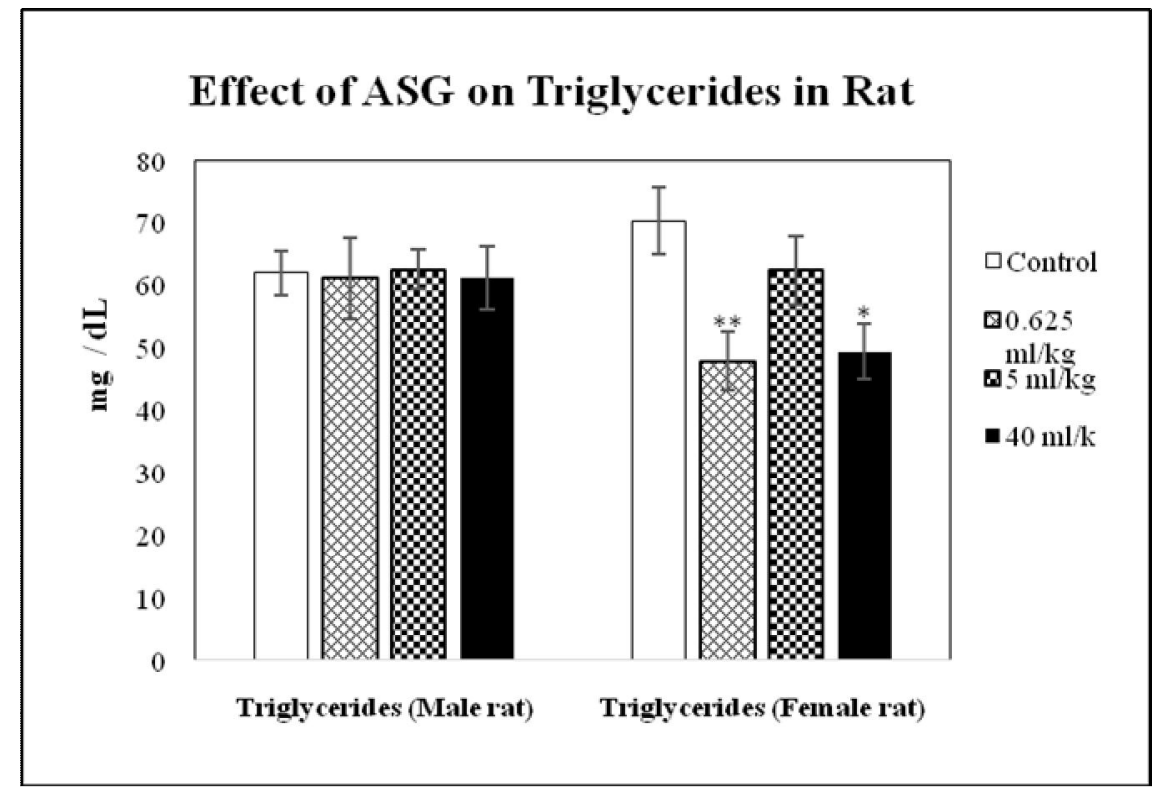

Fig. 4. The effect of ASG on the Serum Triglycerides level utilizing of laboratory animals (Male \& Female Rat) compared to their corresponding Control group after 51 days

Effect on Total Cholesterol: In this study, statistically significant lowering of Total Cholesterol level in ASG treated female rats may be considered as a positive effect. Decreased level of Total Cholesterol level reduces the risk of developing cardiovascular disease. ASG therapy might be beneficial for individuals with risk of cardiovascular disease. The chief ingredient of Ashwagandharishta is the roots of Ashwagandha, Withania somnifera, commonly known for its usefulness in the treatment of hypercholesterolemia, arthritis in combination with other drugs, is also credited to be hypoglycemic and diuretic (Andalu \& Radhika, 2000). Serum cholesterol was also reduced and seated-stature improved in this study (Widodo et al., 2009).

Effect on HDL- Cholesterol: Though the above mentioned results are not statistically significant, it is supported by the following study published previously using rat by utilizing only Withania somnifera (the key plant in Ashwagandharishta preparation.

In that study, hypocholesteremic and antioxidant effects of Withania somnifera (WS) Dunal (Solanaceae) were investigated in hypercholesteremic male albino rats. When the root powder of WS was added to the diet at 0.75 and $1.5 \mathrm{gm} / \mathrm{rat} / \mathrm{day}$, hypercholesteremic animals registered significant increases in serum HDL-cholesterol levels $(+15.10 \%$; $+17.71 \%$ ) were noted in these animals. The significant rise in HDL-C concentration in HWS-I (hypercholesteremic animals administered with $0.75 \mathrm{gm} / \mathrm{rat} / \mathrm{day}$ Withania somnifera root powder) and HWS-II (hypercholesteremic animals administered with 1.5 $\mathrm{gm} / \mathrm{rat} /$ day Withania somnifera root powder) animals as compared to normocholesteraemic subjects indicates the efficacy of WS root powder as a feed 
supplement for controlling body lipid metabolism under hyperlipidemic/hypercholesteremic conditions (Nishant et al., 2007).

To determine risk of developing heart disease and to screen for unhealthy levels of lipids the test for HDL cholesterol (HDL-C) is used along with other lipid tests. Lower levels of blood HDL-cholesterol can increase the risk of developing plaques which may results in atherosclerosis. ASG should be used with caution in those individuals having the risk of atherosclerosis.

Effect on LDL- Cholesterol: For the female rats, a statistically significant decrease was observed in case of LDL cholesterol for the medium and high dose of ASG in comparison to their corresponding control. High LDL cholesterol is a direct risk factor for coronary atherosclerosis. So ASG may decrease the level of risk of atherosclerosis (deposition of excess cholesterol in walls of blood vessel and contributes to hardening of the arteries) and heart disease (Dietschy et al., 1993).

Effect on Triglyceride: The triglyceride level is a laboratory test to measure the amount of triglycerides in blood. Triglycerides are a type of fat and a major source of energy for the body. It is one of the screening tests for excess lipids (fats) in the blood. It is usually part of an evaluation of risk factors for heart disease. Most triglycerides are found in fat (adipose) tissue, but some triglycerides circulate in the blood to provide fuel for muscles to work. Triglycerides move via the blood from the gut to adipose tissue for storage. Most triglycerides are carried in the blood by lipoproteins called very low density lipoproteins (VLDL). A high level of triglycerides in bloodstream is considered a warning sign for cardiovascular health (Semenkovich, 2011).

Conclusion: The present study was limited to just 51 days period on healthy male and female rats. It carries a scope for further evaluation of Ashwagandharishta preparation and also in long term randomized placebo controlled trial to establish its clinical use. Further studies are also required to assess whether the drug can improve other physical parameters or not.

Funding: None

Ethical approval: The present study was approved by the Biosafety, Biosecurity and Ethical Committee of Faculty of Biological Sciences, Jahangirnagar University, Savar, Dhaka-1342, Bangladesh. Reference no BBECJU /M2017 (3)1.

Conflicts of interest: No benefits in any form have been received or will be received from a commercial party related directly or indirectly to the subject of this article.

\section{REFERENCES}

Andallu, B. and Radhika, B. 2000. Hypoglycemic, diuretic and hypocholesterolemic effect of winter cherry (Withania somnifera) root. Indian Journal of Experimental Biology Vol 38 June, pp. 607-609. 
Anonymous. 1992. Bangladesh National Formulary of Ayurvedic Medicine (Approved by the Government of Bangladesh vide Ministry of Health and Family Welfare Memo No. Health-1/Unani-2/89 / (Part - 1) Dhaka, Bangladesh, pp: 13-17.

Dietschy, J.M., Turley, S.D. and Spady, D.K. 1993. Role of liver in the maintenance of cholesterol and low density lipoprotein homeostasis in different animal species.Journal of Lipid Research 34: 1637-1659.

Kushwaha, R. and Karanjekar, S. 2011. Internat. J. Chem. Tech. Res. 3(3), 1033-1036.

Kushwaha, S., Betsy, A. and Chawla, P. 2012. Ethno. Med. 6(2), 111-115.

Nishant, P., Visavadiya, A.V.R.L. Narasimhacharya. 2007. Hypocholesteremic and antioxidant effects of Withania somnifera (Dunal) in hypercholesteremic rats. Phytomedicine: 14, pp; 136-142.

OECD Guideline (425) for the testing of chemicals. 2000. Guidance document on acute oral toxicity, Environmental Health and Safety Monograph Series on Testing and Assessment.

Richmond, W. 1992. Analytical reviews in clinical biochemistry: The quantitative analysis of cholesterol. Annals of Clinical Biochemistry 29: 577-597.

Rifai, N., Warnick, G.R., and McNamara, J.R. 1992. Measurement of low-density-lipoprotein cholesterol in serum: A status report. Clinical Chemistry 38: 150-160.

Semenkovich, C.F. 2011. Disorders of lipid metabolism. In: Goldman L, Ausiello D, eds. Cecil Medicine. 24th ed. Philadelphia, pa: Saunders Elsevier; chap 213.

Sandhu, J. S., Shah, B., Shenoy, S., Chauhan, S., Lavekar, G. S. and Padhi, M. M. 2010. Internat. J. Ayur. Res. 1(3), 144-149.

Tanna, I. R., Aghera, H. B., Ashok, B. K. and Chandola, H. M. 2012. Pharma. Res. 33 (1), 114118.

Widodo, N., Shah, N., Priyandoko, D., Ishii, T., Kaul, S.C. and Wadhwa, R. 2009. Deceleration of senescence in normal human fibroblasts by withanone extracted from ashwagandha leaves. J Gerontol A Biol Sci Med Sci. 64(10):1031-1038. 